\title{
Spontaneous Vesicle Formation from Sodium Salt of Acidic Sophorolipid and Its Application as a Skin Penetration Enhancer
}

\author{
Tomohiro Imura $^{1 *}$, Tomotake Morita ${ }^{1}$, Tokuma Fukuoka', Mizuyuki Ryu², \\ Keisuke Igarashi ${ }^{2}$, Yoshihiko Hirata ${ }^{2}$ and Dai Kitamoto ${ }^{1}$ \\ ${ }^{1}$ Research Institute for Innovation in Sustainable Chemistry, National Institute of Advanced Industrial Science and Technology (AIST) (Tsukuba \\ Central 5-2, Higashi 1-1, Tsukuba, Ibaraki 305-8565, JAPAN) \\ ${ }^{2}$ Biochemical Laboratory, Saraya Co., LTD (24-12, Tmate-cho, Kashiwara, Osaka, 582-0028, JAPAN)
}

\begin{abstract}
In this study, spontaneous vesicle formation from the sodium salt of acidic sophorolipid (SLNa) was observed, and its potential application as a skin penetration enhancer for triterpene glycosides extracted from the fruits of Siraitia grosvenorii Swingle was then investigated. Dynamic light scattering (DLS) measurements of the SLNa assemblies prepared by the gentle mixing of SLNa with water (1\%) showed their hydrodynamic radius $\left(R_{h}\right)$ to be $96.2 \mathrm{~nm}$, and their structure was assigned to be vesicles by freeze-fracture electron microscopy (FF-TEM). DLS and FF-TEM also revealed that the size of the vesicles increased with an increase in the concentration of the triterpene glycosides, indicating that the triterpene glycosides were incorporated into the SLNa vesicles. The results of an in vitro skin permeation assay, after loading the SLNa vesicles on a 3D cultured skin model, showed that the amount of SLNa that penetrated though the skin model increased with time. It was also found that the amount of permeated mogroside, which is the main active component of triterpene glycosides, was significantly enhanced by the SLNa vesicle formulation. These results clearly demonstrated that spontaneously formed vesicles composed of the bolaamphiphile SLNa are useful for application as penetration enhancers for active ingredients such as mogroside $V$.
\end{abstract}

Key words: glycolipid biosurfactant, sophorolipid, spontaneous vesicle, penetration enhancer, triterpene glycosides

\section{INTRODUCTION}

Bio-based surfactants (BSs) are natural amphiphiles that are abundantly produced from a variety of renewable resources by microorganisms. They have received great attention because of their unique properties, including biodegradability, low toxicity, and versatile biological functions, compared to petroleum-based surfactants ${ }^{1-3)}$. The other advantage of BSs is their structural diversity, which is naturally designed and engineered by microorganisms; their unique and complex structures enable them to build up distinct self-assembled structures ${ }^{4,5)}$. Recently, we reported the self-assembly properties of mannosyl erythritol lipids (MELs) produced by the genus Pseudozyma; they self-assembled into giant micelles ${ }^{6)}$, vesicles ${ }^{7)}$, and sponge particles $^{8)}$ depending on the concentration or the functional groups on the head group. Among a variety of assemblies, we have particularly focused on vesicle structures in recent years because they can encapsulate both hydrophobic and hydrophilic compounds, and are known to act as effective skin penetration enhancers ${ }^{9,10)}$. It is also wellknown that conventional vesicles are thermodynamically unstable. Kaler et al. first reported that thermodynamically stable vesicles were spontaneously formed by two synthetic single-tailed surfactants ${ }^{11}$. These vesicles are more stable than conventional vesicles and are easily prepared by gently mixing the two surfactants with water. We also obtained spontaneously formed vesicles by mixing MEL with a phospholipid such as dimyristoyl phosphatidylcholine $(\mathrm{DMPC})^{12)}$. Although the spontaneous vesicles reported so far are generally composed of multicomponent synthetic surfactant systems, some bolaform surfactants having two hydrophilic groups at both ends of a hydrocarbon chain generated unique spontaneous vesicles composed of monolayers and not bilayers ${ }^{13,14)}$.

\footnotetext{
*Correspondence to: Tomohiro Imura, Research Institute for Innovation in Sustainable Chemistry, National Institute of Advanced Industrial Science and Technology (AIST) (Tsukuba Central 5-2, Higashi 1-1, Tsukuba, Ibaraki 305-8565, JAPAN)

E-mail: t-imura@aist.go.jp

Accepted October 13, 2013 (recieved for review July 28, 2013)

Journal of Oleo Science ISSN 1345-8957 print / ISSN 1347-3352 online

http://www.jstage.jst.go.jp/browse/jos/ http://mc.manusriptcentral.com/jjocs
} 
On the other hand, sophorolipids (SLs) are promising glycolipids BSs, generally produced by Starmerella bombicola $^{15)}$. They exhibit considerably high productivity with yields over $100 \mathrm{~g} / \mathrm{L}$ from different carbon sources, and are efficiently recovered from culture broths, mainly by precipitation ${ }^{16)}$. Unlike other BSs, SLs are produced as a mixture of acid- and lactone-type sophorolipids. Because of this, SLs display a high detergency, ready biodegradability, low foaming, and low cytotoxicity ${ }^{17}$. Furthermore, they are also characterized by high tolerance to hard water and have been used as commercial detergents in households and healthcare facilities in Japan ${ }^{18,19)}$. SLs and their derivatives have also been found to display anti-microbial ${ }^{20)}$, anticancer $^{21)}$, and anti-virus activities ${ }^{22)}$. Thus, they are of growing commercial interests in the cosmetics, food, and detergent industries. Of all the SL homologues, acidic SL is an asymmetric bolaform structure with two different hydrophilic groups (Fig. 1). The disaccharide polar head and carboxyl group provide many sites for intermolecular association through hydrogen bonding.

Siraitia grosvenori Swingle (SG) is a traditional Chinese fruit, and a herbaceous perennial vine of the Cucurbitaceae family. SG has been used as a folk medicine in China for cough, sputum, asthma, bronchitis, pharyngitis, acute gastritis, and constipation. The most remarkable characteristic of $\mathrm{SG}$ is its unique property of sweetness. The chemical structure of the sweetening component in SG has been identified to belong to the triterpenoid named as mogroside for various glycosylated compounds and as mogrol for aglycones $^{23)}$. The sweetening components in this plant include mogroside $\mathrm{V}$ and 11-oxo-mogroside $\mathrm{V}$; tetraglucose conjugated mogrosides include mogroside IV and siamenoside I, and triglucose conjugated mogrosides include mogroside III. Previous studies on the pharmacological activities of SG extracts revealed anti-atherosclerotic effects ${ }^{24)}$, anti-cancer activity $^{25,26)}$, anti-allergy activity ${ }^{27)}$, and anti-diabetic effects $^{28,29)}$ in animal models. Thus, they are of growing commercial interests in cosmetic industry.

In this study, we report the spontaneous vesicle formation from the sodium salt of acidic SLs (SLNa) for the first time, and their characterization using dynamic light scattering (DLS) and freeze-fracture electron microscopy (FF-

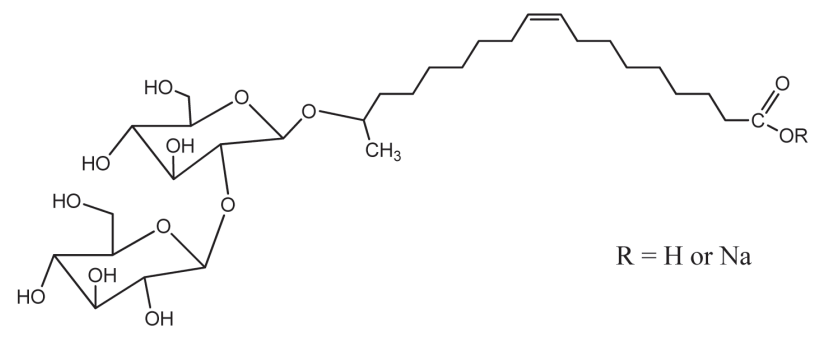

Fig. 1 The structure of acidic form of sophorolipid (SLH) and its sodium salt (SLNa).
TEM). Moreover, SL vesicles including triterpene glycosides extracted from the fruits of SG were newly prepared, and their penetration ability though a 3D cultured human skin model was evaluated by an in vitro penetration assay.

\section{EXPERIMENTAL}

\subsection{Microorganism and culture conditions}

The SL mixture was microbially produced by the following procedure ${ }^{30}$ : a seed culture $(1.5 \mathrm{~mL})$ of the yeast strain of Starmerella bombicola NBRC 10243 was transferred to a $300 \mathrm{~mL}$ Erlenmeyer flask containing $30 \mathrm{~mL}$ of a fermentation medium [1\% (w/v) glucose, $0.5 \%(\mathrm{w} / \mathrm{v})$ peptone, $0.3 \%$ $(\mathrm{w} / \mathrm{v})$ yeast extract, $0.3 \%(\mathrm{w} / \mathrm{v})$ malt extract, and distilled water], followed by incubation at $30{ }^{\circ} \mathrm{C}$ on a rotary-shaker $(300 \mathrm{rpm})$. The seed cultures were transferred into a minijar fermenter(TBR-2-3, Sakura S. J., Tokyo) with 2.5 L fermentation medium [10\% (w/v) soybean oil, $10 \%(\mathrm{w} / \mathrm{v})$ glucose, $0.25 \%(\mathrm{w} / \mathrm{v})$ yeast extract, $0.1 \%(\mathrm{w} / \mathrm{v})$ urea, $0.5 \%$ $(\mathrm{w} / \mathrm{v}) \mathrm{MgSO}_{4} \cdot 7 \mathrm{H}_{2} \mathrm{O}, 0.1 \%(\mathrm{w} / \mathrm{v}) \mathrm{NaCl}$, and $\left.1 \%(\mathrm{w} / \mathrm{v}) \mathrm{KH}_{2} \mathrm{PO}_{4}\right]$. The cultivation was performed at $30{ }^{\circ} \mathrm{C}, 500 \mathrm{rpm}$, and 1 vvm of aeration for 7 days.

\subsection{Purification and alkaline hydrolysis of the SLs.}

The culture broth was then extracted with an equal volume of ethyl acetate. The organic layer was separated and evaporated. The resulting yellow oil (50 g) was dissolved in chloroform and placed on a column $(5 \times 40 \mathrm{~cm})$. The mixture of SL was then chromatographed with a close gradient elution of chloroform - acetone ( $10: 0$ to $0: 10)$. Alkaline hydrolysis of the SLs mixture led to the deacetylated acidic structures. The SLs mixture $(1.0 \mathrm{~g})$ was dissolved in $0.5 \mathrm{~mL}$ of $5 \mathrm{~N} \mathrm{NaOH}(\mathrm{aq})$ and placed in a test tube, and then the test tube was tightly sealed with a screw cap and heated at $80{ }^{\circ} \mathrm{C}$ in a water bath for $1 \mathrm{~h}$. After cooling, the reaction medium was carefully acidified to $\mathrm{pH} 1.0$ by the dropwise addition of $\mathrm{HCl}(\mathrm{aq})$ and then centrifuged (9000 rpm, $5 \mathrm{~min}$ ). The upper layer was then removed, and the lower layer including the acidic SL was washed with water three times. The acidic SL was neutralized by the addition of $3 \mathrm{~N} \mathrm{NaOH}$. SLNa was obtained by freezedrying. The structure was assigned by ${ }^{1} \mathrm{H}-\mathrm{NMR}$ and MALDITOF-MS as previously reported ${ }^{311}$.

\subsection{Dynamic Light Scattering}

The size of the assembled structures was measured with a DLS-7000 (Otsuka Electronics Co., Japan) using a He-Ne laser of $633 \mathrm{~nm}$ wavelength as a light source at $25^{\circ} \mathrm{C}$. Triterpene glycosides extracted from the fruits of SG were kindly supplied from Saraya Co., Ltd. (Osaka, Japan).

The time-dependent correlation function of the scattered light intensity was measured at a scattering angle of $90^{\circ}$. The DLS intensity data were processed using the in- 
strumental software to obtain the hydrodynamic diameter, polydispersity index, and mass diffusion coefficient of the samples. The mass diffusion coefficient $D$ was derived from the decay time $\left(\tau_{\mathrm{C}}\right)$ of the intensity correlation function as $D=\left(2 k_{L}{ }^{2} \tau_{C}\right)^{-1}$, where $k_{L}$ is the scattering wave vector. The hydrodynamic mass diffusion coefficient $D_{0}$ was obtained as the limit of $D$ as $k_{L}$ goes to zero. $D_{0}$ was found to obey the Stokes-Einstein relation, $D_{0}=k T / 6 \pi \eta R_{H}$, where $k$ is the Boltzmann constant, $T$ is the absolute temperature, $\eta$ is the viscosity of the solution, and $R_{H}$ is the hydrodynamic radius.

\subsection{Freeze-Fracture Electron Microscopy}

FF-TEM was used to determine the structure of the assemblies. Some samples were frozen with liquid nitrogen at $-189{ }^{\circ} \mathrm{C}$. The fracture process was performed with a JFD-9010 (JOEL, Japan) at $-130{ }^{\circ} \mathrm{C}$, and the fractured surface was then replicated by evaporating the platinum at an angle of $60^{\circ}$, followed by carbon at an angle of $90^{\circ}$ to strengthen the replica. The replicate was placed on a 400 mesh copper grid after being washed with water, methanol, and chloroform. It was then examined and photographed using a JEM-1010 (JOEL, Japan) transmission electron microscope.

\subsection{Determination of the surface activity}

The surface tension of SLH and SLNa was determined by the pendant drop method at $25{ }^{\circ} \mathrm{C}$, which was performed using an apparatus consisting of an automatic interfacial tension meter(DM500, Kyowa Interface Science, Japan) and the Drop Shape Analysis software of FAMAS ver. 2.01. The drop was formed at the tip of the syringe by pressing the solution out by means of a setscrew. The drop shape analysis was performed as follows: a drop profile was extracted from the drop image; then, a curve fitting program compared the experimental drop profile with a theoretical one (Young-Laplace method) and gave the corresponding surface tension value. For each concentration of the purified glycolipid solution, the evolution of the drop surface tension was followed over $30 \mathrm{~min}$.

\subsection{In vitro skin permeation study}

The transdermal absorption of SLNa and mogroside V, which is an effective ingredient of SG, was measured using the Upright Franz cell assembly (PermeGear Inc., Hellertown, Pa.). TESTSKIN LSE-high, purchased from Toyobo Co., Ltd. (Osaka, Japan), was mounted between the donor and receptor compartments. The donor consisted of 1.0 $\mathrm{mL}$ of the vehicle containing either $\mathrm{SG}(0.1 \%)$ alone or $\mathrm{SG}$ $(0.1 \%)$ with $\mathrm{SLNa}(1 \%)$. The receptor medium $(4.5 \mathrm{~mL})$ was phosphate-buffered saline (PBS, pH 7.2). The available diffusion area between compartments was $0.64 \mathrm{~cm}^{2}$. The stirring rate was kept constant and the temperature of the receptor was mainlined at $37^{\circ} \mathrm{C}$. At 30,60 , and $120 \mathrm{~min}$,
$300 \mu \mathrm{L}$ aliquots of the receptor medium were withdrawn for the quantitation of the SLs or mogroside V. The accumulated amounts of SLs and mogroside $\mathrm{V}$ were determined by RP-HPLC as described previously ${ }^{28,29)}$.

\section{RESULTS}

\subsection{Surface activity of SLH and SLNa}

The lowering of the surface tension is the most important physical property of surfactants, and the surface tension in aqueous solution was measured by the pendant drop method at $25{ }^{\circ} \mathrm{C}$. Figure 2 shows the surface tension plotted as a function of SLH or SLNa concentration. The surface tensions of both decreased with an increase in the concentration and became constant at their critical aggregation concentrations (CAC). The CAC and $\gamma \mathrm{CAC}$ were determined by the intersection of two fitted lines, and the estimated CAC and $\gamma \mathrm{CAC}$ for SLH were $0.034 \%$ and $35.3 \mathrm{mN} /$ $\mathrm{m}$, which agree well with the values reported in the literature $^{30)}$. The CAC and $\gamma$ CAC of the sodium salt of SL, SLNa, were $0.083 \%$ and $32.4 \mathrm{mN} / \mathrm{m}$, indicating that SLNa can lower the surface tension more effectively than SLH. The higher hydrophilicity of SLNa resulted in its higher CAC value. Because we found that SLNa possesses a high surface activity and a high aqueous solubility, SLNa was used for further experiments.

\subsection{SLNa assemblies including triterpene glycosides characterized by DLS and FF-TEM}

The structure of the SLNa assemblies above the CAC and the effect of the addition of triterpene glycosides on

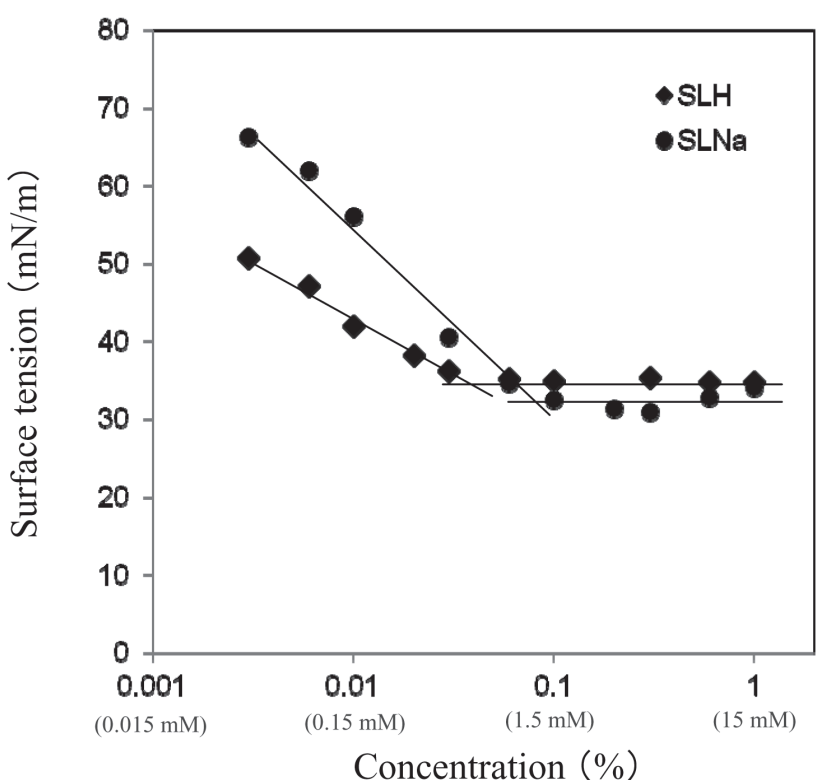

Fig. 2 Relationship between surface tension and surfactant concentration. SLH OSLNa. 
the structure were investigated by DLS and FF-TEM. The triterpene glycosides extracted from the fruits of SG are potentially useful as important sources of pharmaceutical and sweetener compounds. They are known to be about a hundred times sweeter than sucrose but low in calories ${ }^{23)}$. Mogroside V, which is one of the major components, inhibits the initiation and promotion of cancer, and exhibits antioxidant and antiallergic effects ${ }^{35)}$.

The hydrodynamic radii $\left(\mathrm{R}_{\mathrm{h}}\right)$ of the SLNa assemblies measured by DLS are shown in Table 1 . The $R_{h}$ of the pure SLNa assemblies at a concentration of $1 \%$ was $96.2 \mathrm{~nm}$, and the $R_{h}$ increased with an increase in the triterpene glycosides concentration. Zhou et al. reported on self-assembling structures of SLNa in dilute solutions $(<0.17 \%)^{4)}$, where SLNa self-assembles into giant aggregates whose $R_{h}$ were below $100 \mathrm{~nm}$; the size was found to dependent on the concentration. However, the detailed structure of the SLNa assemblies is still unclear because they used only DLS to determine the structure.

To address this, we directly observed the SL assemblies by FF-TEM. Figure $3(a)$ shows the FF-TEM images of the SL assemblies at a concentration of $1 \%$. The image clearly indicates a vesicle structure whose size is similar to that predicted by the DLS measurements. Interestingly, Guilbot et al. reported the spontaneous formation of vesicles composed of unsymmetrical bolaamphiphiles bearing a neutral glycosidic polar and an electropositive ammonium group ${ }^{13)}$. Chen et al. also reported thermodynamically stable vesicles formed with orotic acid-derived bolaamphiphiles ${ }^{14)}$. The

Table 1 The effect of addition of triterpene glycosides on particle size of SLNa assemblies.

\begin{tabular}{cc}
\hline Triterpene glycosides & Particle size $(\mathrm{nm})$ \\
\hline $0 \%$ & 96.2 \\
$0.02 \%$ & 114.7 \\
$0.1 \%$ & 182.6 \\
\hline
\end{tabular}

a)

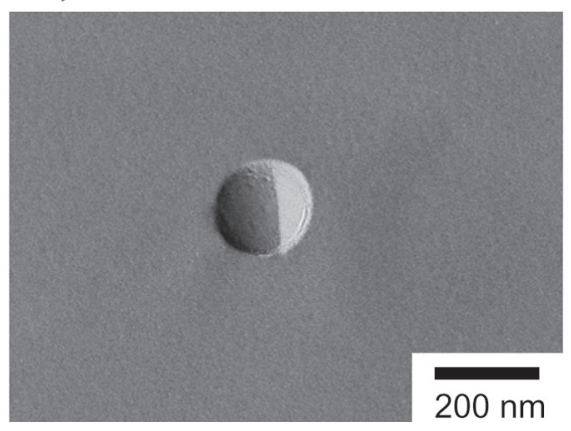

b)

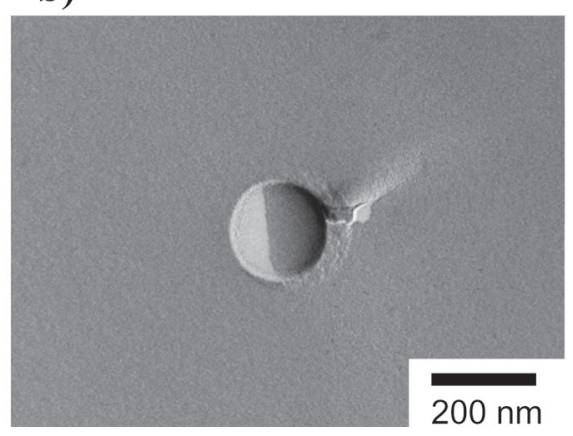

observed SLNa vesicles were spontaneously formed by gently mixing with water, and the SLNa vesicle solution was stable for over one month at room temperature, demonstrating the formation of thermodynamically stable vesicles probably composed not of an ordinary bilayer but a monolayer structure.

Moreover, based on both the DLS and FF-TEM measurements, the size of the SLNa vesicles increased with an increase in the concentration of triterpene glycosides extracted from the fruits of SG, suggesting that the formation of the SL vesicles included the triterpene glycosides, even though the solubilization locus of the triterpene glycosides in the SLNa vesicles is unclear. These results clearly demonstrated that thermodynamically stable vesicles including triterpene glycosides were prepared for the first time by using a naturally derived bolaamphiphile bearing sophorose and electronegative carboxylate groups at both ends.

\subsection{In vitro skin permeation study}

To enhance the transdermal absorption of active ingredients, vesicle formulations have recently attracted atten$\operatorname{tion}^{9,10)}$; vesicles, often called liposomes, are known to penetrate well when their size is about $300 \mathrm{~nm}^{36}$. Even though most vesicles reported as penetration enhancers have been conventional vesicles composed of bilayer membranes, in this study, the thermodynamically stable vesicles formed by the bolaform amphiphile SLNa were used as a penetration enhancer for triterpene glycosides extracted from the fruits of SG.

For the skin permeation study, a 3D cultured human skin model, TESTSKIN LSE-high, was used. It consists of a multilayer of stratum corneum (SC), viable epidermis, and a dermis for which skin fibroblasts are embedded in a collagen gel. The skin properties are close to those of biogenic skin, and the characteristics of the in vitro skin penetration correlate well with those of the in vivo situation ${ }^{37}$.

The amount of permeation of SLNa vesicles though the skin was investigated first. SLNa vesicles (1\%) without triterpene glycosides were applied to the test skin, where the

Fig. 3 Freeze-fracture electron micrographs of SLNa assemblies including triterpene glycosides. a) $0 \%$ b) $0.02 \%$ c) $0.1 \%$ (Triterpene glycosides conc). 


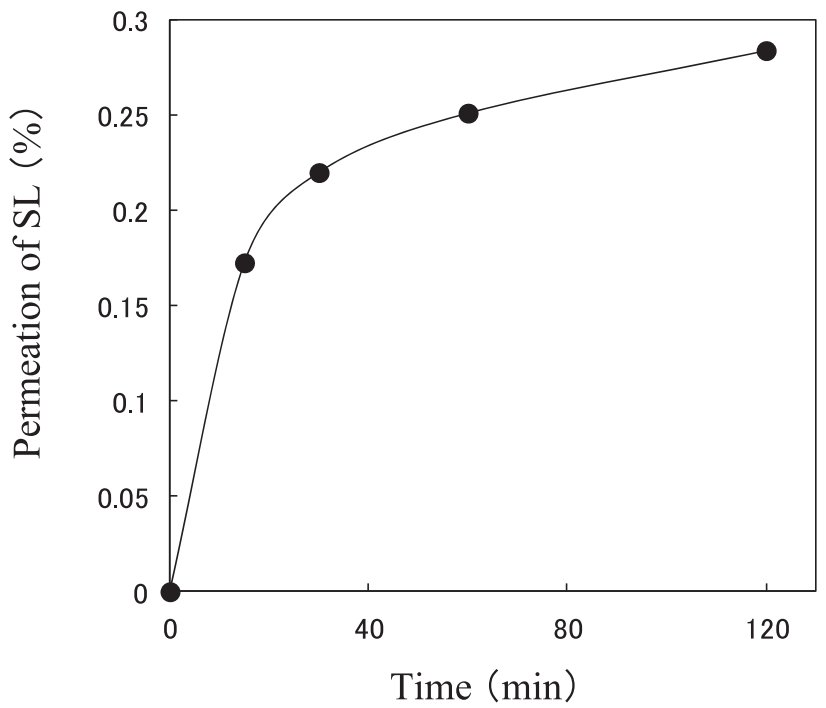

Fig. 4 Time dependence of transdermal absorption of SLNa having vesicle structure without triterpene glycosides.

vesicle size was $96.2 \mathrm{~nm}$, as described in Table 1. Figure 4 shows the time dependence of the amount of SLNa permeated through the skin. The figure clearly indicates that the amount of penetrated SLNa increased with an increase in time, suggesting that SLNa could act as a penetration enhancer. We further investigated the effect of the SLNa vesicle formulation on the permeation of triterpene glycosides extracted from the fruits of SG. The SLNa vesicles containing $0.1 \%$ triterpene glycosides were prepared and loaded on the test skin. The size of the vesicles was 182.6 $\mathrm{nm}$, as described in Table 1. Among the triterpene glycosides, the amount of permeated mogroside $\mathrm{V}$, which is one of the major active and representative components and

(a)

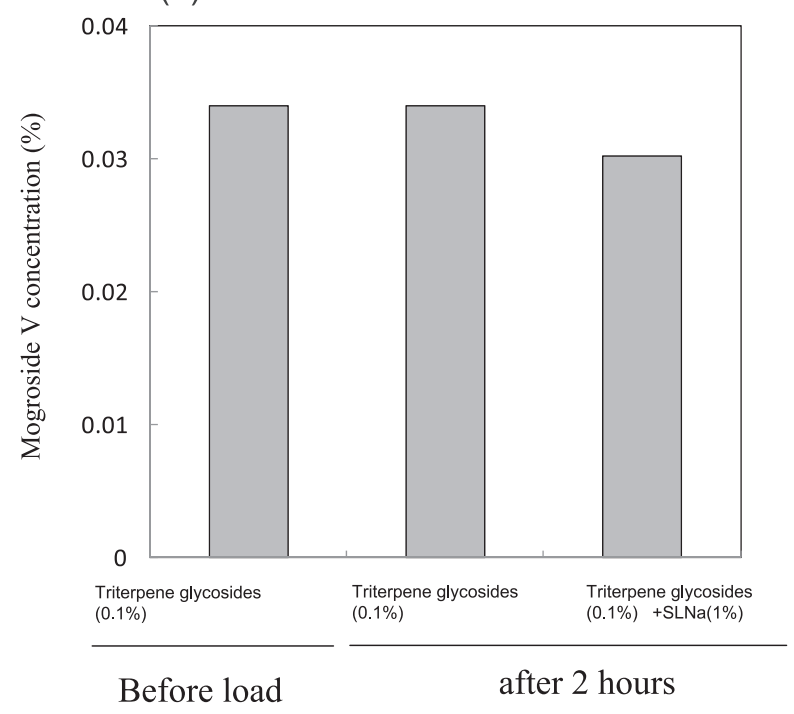

water-soluble, was determined by HPLC, and the results are shown in Fig. 5(a). The structure of mogroside $\mathrm{V}$ is shown in Fig. 5(b). As shown in the figure, the amount of permeated mogroside $\mathrm{V}$ increased by the SLNa vesicle formulation after $2 \mathrm{~h}$. This clearly demonstrates that the SLNa vesicles could act as a penetration enhancer for active ingredients.

Recently, Ishii et al. reported that vesicle-like structures composed of the acid form of sophorolipid, whose size ranges from 40 to $2700 \mathrm{~nm}$, also act as a penetration enhancer for lactoferrin ${ }^{38)}$. Compared with their system, the vesicles we prepared were much smaller and had a narrower size distribution. Thermodynamically stable vesicles are also known to be easily prepared compared with typical vesicles. Although the detailed mechanism of the penetration enhancement is still unclear, and further studies including in vivo experiments are necessary to clarify the mechanism, the spontaneously formed vesicles composed of bolaamphiphiles presented herein would be useful biomaterials for exploring transdermal applications. We will continue with further detailed studies to clarify the mechanism of the penetration enhancement.

\section{CONCLUSION}

Spontaneous vesicle formation from the bolaform amphiphile, SLNa, was observed, and the potential application of the vesicles as a skin penetration enhancer for triterpene glycosides extracted from the fruits of SG was then investigated. DLS measurements of the SLNa assemblies prepared by gently mixing SLNa with water (1\%) revealed their hydrodynamic radius to be $96.2 \mathrm{~nm}$, and their structure was determined to be vesicles using FF-TEM.

(b)

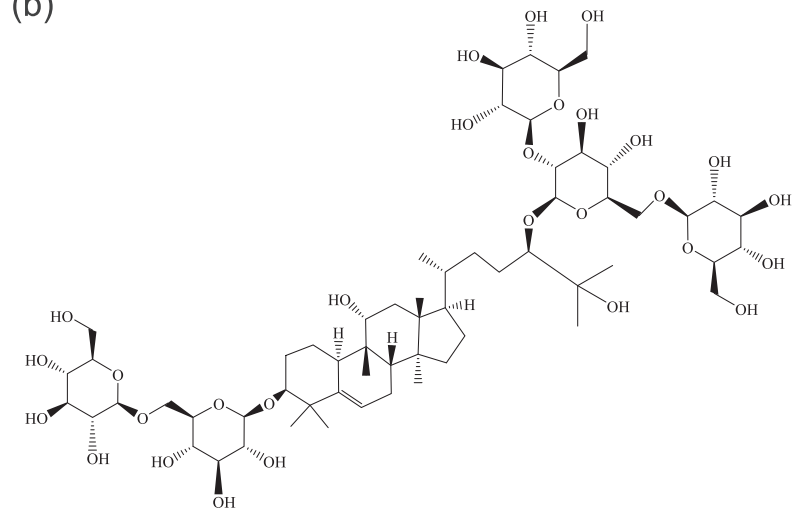

Fig. 5 (a) Transdermal absorption of mogroside V with or without SLNa assemblies. (b) Structure of mogroside V. 
DLS and FF-TEM measurements also revealed that the size of vesicles increased with an increase in the concentration of triterpene glycosides, indicating that the triterpene glycosides were incorporated into the SLNa vesicles. An in vitro skin permeation assay, after loading the SLNa vesicles on a 3D cultured skin model, showed that the amount of SLNa that penetrated though the skin model increased with time. It was also found that the amount of permeated mogroside, which is the main active component of triterpene glycosides, was significantly enhanced by the SLNa vesicle formulation.

Our results clearly demonstrated that spontaneously formed SLNa vesicles are useful as a penetration enhancer for active ingredients such as mogroside $\mathrm{V}$.

\section{References}

1) Banat, I. M.; Makkar, R. S.; Cameotra, S. S. Potential commercial applications of microbial surfactant. Appl. Microbiol. Biotechnol. 53, 495-508(2000).

2) Kitamoto, D.; Isoda, H.; Nakahara, T. Functions and potential applications of glycolipid biosurfactants: from energy-saving materials to gene delivery carriers. J. Biosci. Bioeng. 94, 187-201(2002).

3) Mulligan, C. N. Environmental applications for biosurfactants. Environ. Pollut. 133, 183-198(2005).

4) Zhou, S.; Xu, C.; Wang, J.; Gao, W.; Akhverdiyeva, R.; Shah, V.; Gross, R. Supramolecular Assemblies of a Naturally Derived Sophorolipid. Langmuir 20, 79267932 (2004).

5) Imura, T.; Kawamura, D.; Ishibashi, Y.; Morita, T.; Sato, S.; Fukuoka, T.; Kikkawa, Y.; Kitamoto, D. Low molecular weight gelators based on biosurfactants, cellobiose lipids by Cryptococcus humicola. J. Oleo Sci. 61(11), 659-664 (2012).

6) Imura, T.; Ohta, N.; Inoue, K.; Yagi, N.; Negishi, H.; Yanagishita, H.; Kitamoto, D. Naturally Engineered Glycolipid Biosurfactants Leading to Distinctive SelfAssembled Structures. Chem. Eur. J. 12, 2434-2440 (2006).

7) Worakitkanchanakul, W.; Imura, T.; Fukuoka, T.; Morita, T.; Sakai, H.; Abe, M.; Rujiravanit, R.; Chavadej, S.; Minamikawa, H.; Kitamoto, D. Aquous-phase behavior and vesicle formation of natural glycolipid biosurfactant, mannosylerythritol lipid-B. Colloids Surf. B 65, 106-112 (2008).

8) Imura, T.; Yanagishita, H.; Kitamoto, D. Coacervate formation from natural glycolipid: One acetyl group on the headgroup triggers coacervate-to-vesicle transition. J. Am. Chem. Soc. 126, 10804-10805 (2004).

9) Duangjit, S.; Obata, Y.; Sano, H.; Kikuchi, S.; Onuki, Y.; Opanasopit, P.; Ngawhirunpat, T.; Maitani, Y.; Takayama, K. Menthosomes, novel ultradeformable vesicle for transdermal drug delivery: optimization and characterization. Biol. Pharm. Bull. 35(10), 1720-1728 (2012).

10) Pierre, M. B. R.; Costa, I. S. M. Liposomal systems as drug delivery vehicles for dermal and transdermal applications. Arch Dermatol Res 303, 607-621 (2011).

11) Kaler, E. W.; Murthy, A. K.; Rodriguez, B. E.; Zasadzinski, J. A. N. Spontaneous vesicle formation in aqueous mixtures of single-tailed surfactants. Science 245, 1371-1374(1989).

12) Imura, T.; Yanagishita, H.; Ohira, J.; Sakai, H.; Abe, M.; Kitamoto, D. Thermodynamically stable vesicle formation from glycolipid biosurfactant sponge phase. Colloids Surf. B 43, 115-121 (2005).

13) Guilbot, J.; Benvegnu, T.; Legros, N.; Plusquellec, D. Efficient synthesis of unsymmetrical bolaamphiphiles for spontaneous formation of vesicles and disks with a transmembrane organization. Langmuir 17, 613-618 (2001).

14) Chen, Z. X.; Su, X. X.; Deng, S. P. Molecular recognition of melamine by vesicles spontaneously formed from orotic acid derived bolaamphiphiles. J. Phys. Chem. 115, 1798-1806 (2011).

15) Ashby, R. D.; Solaiman, D. K. Y.; Foglia, T. A. The use of Fatty acid esters to enhance free acid sophorolipid synthesis. Biotechnol. Lett. 28, 253-260 (2006).

16) Van Bogaert, I. N. A.; Saerens K.; Muynck, C. D.; Develter, D.; Soetaert, W.; Vandamme, E. J. Microbail production and application of sophorolipids. Appl. Microbiol. Biotechnol. 76, 23-34(2007).

17) Hirata, Y., Ryu, M., Oda, Y., Igarashi, K., Nagatsuka, A., Furuta, T, and Sugiura, M.: Novel characteristics of sophorolipids, yeast glycolipids biosurfactants, as biodegradable low-foaming surfactants. J. Biosci. Bioeng., 108, 142-146(2009).

18) Hirata, Y., Ryu, M., Igarashi, K., Nagatsuka, A., Furuta, T, Kanaya, S., and Sugiura, M.: Natural synergism of acid and lactone type mixed sophorolipids in interfacial activities and cytotoxicity. J. Oleo Sci., 58, 565572 (2009).

19) Hirata, Y., Ito, H., Furuta, T., Ikuta, K., and Sakudo, A.: Degradation and destabilization of abnormal prion protein using alkaline detergents and proteases. Int. J. Mol. Med., 25, 267-270 (2010).

20） Kim, H.-S.; Kim, Y.-B.; Lee, B.-S.; Kim, E.-K. Sophorolipid production by Candida bombicola ATCC 22214 from a corn-oil processing by product. J. Microbiol. Biotechnol. 15, 55-58(2005).

21) Chen, J.; Song. X.; Zhang, H.; Qu, Y.; Mario, J.-Y. Sophorolipid produced from the new yeast strain Wickerhamiella demercqiae induces apoptosis in $\mathrm{H} 7402 \mathrm{hu}-$ man liver cancer cells. Appl. Microbiol. Biotechnol. 72, 52-59(2006).

22) Azim, A.; Shah, V.; Doncel, G. F.; Peterson, N.; Gao, W.; 
Gross, R. Amino acid conjugated sophorolipids: A New Family of Biologically Active Functionalized Glycolipids. Bioconjug. Chem. 17, 1523-1529(2006).

23) Kasai, R.; Nie, R. L.; Nashi, K.; Otani, K. Sweet cucurbitane glycosides from fruits of Siraitha siamensis a Chinese folk medicine. Agric. Biol. Chem. 53, 33473349 (1989).

24) Takeo, E.; Yoshida, H.; Tada, N.; Shingu, T.; Matsuura, H.; Murata, Y.; Yoshikawa, S.; Ishikawa, T.; Nakamura, H.; Ohsuzu, F.; Kohda, H. Sweet elements of Siraitia grosvenori inhibit oxidative modification of low-density lipoprotein. J. Atherosclerosis Thromb. 9(2), 114120(2002)

25) Takasaki, M.; Konoshima, T.; Murata, Y.; Sugiura, M.; Nishino, H.; Tokuda, H.; Matsumoto, K.; Kasai, R.; Yamasaki, K. Anticarcinogenic activity of natural sweeteners, cucurbitane glycosides, from Momordica grosvenori. Cancer Lett. 198, 37-42 (2003).

26) Yasuno, H.; Nishimura, J.; Dewa, Y.; Muguruma, M.; Takabatake, M.; Murata, Y.; Shibutani, M.; Mitsumori, K. Modifyling effect of Siraitia grosvenori extract on piperonyl butoxide-promoted hepatocarcinogenesis in rats. J. Toxicol. Sci. 33, 197-207 (2008).

27) Hossen, MA.; Shinmei, Y.; Jiang, S.; Takubo, M.; Tsumuro, T.; Murata, Y.; Sugiura, M.; Kamei, C. Effect of Lo Han Kuo (Siraitia grosvenori Swingle) on nasal rubbing and scratching behavior in ICR mice. Biol. Pharm. Bull. 28, 238-241 (2005).

28) Suzuki, YA.; Murata, Y.; Inui, H.; Sugiura, M.; Nakano, Y. Triterpene glycosides of Siraitia grosvenori inhibit rat intestinal maltase and suppress the rise in blood glucose level after a single oral administration of maltose in rats. J. Agric. Food Chem. 53, 2941-2946 (2005).

29) Suzuki, YA.; Tomodo, M.; Murata, Y.; Inui, H.; Sugiura, M.; Nakano, Y. Antidiabetic effect of long-term supplementation with Siraitia grosvenori on the spontaneously diabetic Goto-Kakizaki rat Br. J. Nutr., $97(4)$, 770-775 (2007).

30) Zhou, Q. H.; Klekner, V.; Kosaric, N. Production of sophorose lipids by Torulopsis bombicola from safflower oil and glucose. J. Am. Oil Chem. Soc., 69,
89-91 (1992).

31) Imura, T.; Masuda, Y.; Minamikawa, H.; Fukuoka, T.; Konishi, M.; Morita, T.; Sakai, H.; Abe, M.; Kitamoto, D. Enzymatic conversion of diacetylated sophoroselipid into acetylated glucoselipid: Surface-active properties of novel bolaform biosurfactants. J. Oleo Sci. 59(9), 495-501 (2010).

32) Murata, Y.; Ogawa, T.; Suzuki, YA.; Yoshikawa, S.; Inui, H.; Sugiura, M.; Nakano, Y. Digestion and absorption of siraitia grosvenori triterpenoids in the rat. Biosci. Biotechnol. Biochem. 74(3), 673-676(2010).

33) Konishi, M.; Fukuoka, T.; Morita, T.; Imura, T.; Kitamoto, D. Production of New Types of Sophorolipids by Candida batistae. J. Oleo Sci. 57(6), 359-369 (2008).

34) Chen, M.; Dong, C.; Penfold, J.; Thomas, R. K.; Smyth, T. J. P.; Perfumo, A.; Marchant, R.; Banat, I. M.; Stevenson, P.; Parry, A.; Tucker, I.; Campbell, R. A. Adsorption of sophorolipid biosurfactants on their own and mixed with sodium dodecyl benzene sulfonate, at the air/water interface. Langmuir 27, 8854-8866 (2011).

35) Konoshima, T.; Takasaki, M. Cancer-chemopreventive effects of natural sweeteners and related compounds. Pure Appl. Chem. 74, 1309-1316(2002).

36) DuPlessis, E. R.; Rohlfs, R. J.; Hille, R.; Thorpe, C. Electron-transferring flavoprotein from pig and the methylotrophic bacterium W3A1 contains AMP as well as FAD. Biochem. Mol. Biol. Int. 32 (1), 195-199 (1994).

37) Watanabe, T.; Hasegawa, T.; Takahashi, H.; Ishibashi, T.; $\mathrm{K}, \mathrm{S}$. Effect of penetration enhancers on the permeation of drugs across a three-dimensional cultured human skin model: comparison with the effects using excised hairless rat skin. Altern. Animal Test Experiment. 8, 15-22(2001).

38) Ishii, N.; Kobayashi, T.; Matsumiya, K.; Ryu, M.; Hirata, Y.; Matsumura, Y.; Suzuki, Y. A. Transdermal administration of lactoferrin with sophorolipid. Biochem. Cell Biol. 90, 504-512(2012). 